\title{
Article \\ Context-Adaptive Availability Notifications for an SAE Level 3 Automation
}

\author{
Simon Danner*, Alexander Feierle $\mathbb{D}^{\text {, Carina Manger and Klaus Bengler }}$ \\ Chair of Ergonomics, Department of Mechanical Engineering, Technical University Munich, \\ 85747 Garching, Germany; alexander.feierle@tum.de (A.F.); carina.manger@tum.de (C.M.); \\ bengler@tum.de (K.B.) \\ * Correspondence: simon.danner@tum.de
}

Citation: Danner, S.; Feierle, A.;

Manger, C.; Bengler, K.

Context-Adaptive Availability

Notifications for an SAE Level 3

Automation. Multimodal Technol.

Interact. 2021, 5, 16. https://

doi.org $/ 10.3390 / \mathrm{mti} 5040016$

Academic Editors: Shadan Sadeghian Borojeni and Philipp Wintersberger

Received: 1 February 2021

Accepted: 24 March 2021

Published: 29 March 2021

Publisher's Note: MDPI stays neutral with regard to jurisdictional claims in published maps and institutional affiliations.

Copyright: (C) 2021 by the authors Licensee MDPI, Basel, Switzerland This article is an open access article distributed under the terms and conditions of the Creative Commons Attribution (CC BY) license (https:// creativecommons.org/licenses/by/ $4.0 /)$

\begin{abstract}
Context-adaptive functions are not new in the driving context, but even so, investigations into these functions concerning the automation human-machine interface (aHMI) have yet to be carried out. This study presents research into context-adaptive availability notifications for an SAE Level 3 automation in scenarios where participants were surprised by either availability or non-availability. For this purpose, participants $(N=30)$ took part in a driving simulator study, experiencing a baseline HMI concept as a comparison, and a context-adaptive HMI concept that provided context-adaptive availability notifications with the aim of improving acceptance and usability, while decreasing frustration (due to unexpected non-availability) and gaze deviation from the road when driving manually. Furthermore, it was hypothesized that participants, when experiencing the context-adaptive HMI, would activate the automated driving function more quickly when facing unexpected availability. None of the hypotheses could be statistically confirmed; indeed, where gaze behavior was concerned, the opposite effects were found, indicating increased distraction induced by the context-adaptive HMI. However, the trend in respect to the activation time was towards shorter times with the context-adaptive notifications. These results led to the conclusion that context-adaptive availability notifications might not always be beneficial for users, while more salient availability notifications in the case of an unexpected availability could be advantageous.
\end{abstract}

Keywords: automated driving; driving-simulator study; availability; HMI; context adaptivity

\section{Introduction}

The introduction of automated driving functions of SAE Level 3 [1] is associated with different expectations; for instance, less congestion, improved fuel efficiency, increased traffic safety, and the possibility of conducting non-driving related activities (NDRAs) [2,3]. While these expectations are all positive, there are also concerns regarding automated driving, such as liability concerns, safety and privacy concerns, and the fear of system errors [2]. In an exploratory study, participants were asked what reasons they would have for activating an automated driving system if available and what reasons they would have for not activating it. The main reason for activation appeared to be the possibility of conducting NDRAs, while the main reason for non-activation was reported to be the possibility of driving faster than an automated driving system (ADS) would [4]. As SAE Level 3 automated vehicles are not yet on the market, people cannot state which NDRAs they actually conduct, but research has provided answers regarding what kind of NDRAs will probably be conducted in the future. Firstly, the activities carried out today while driving manually will probably still be conducted while driving automatically [5]. Moreover, activities such as reading, watching movies, working, browsing on a smart phone, and others are thought to be likely [5-7]. If a user is able to conduct an NDRA for at least as long as was planned before starting it, the activation of the automation system can be defined as purposeful [4,8]. It was shown that in order to be able to use an automated driving function purposefully, information on the availability of the ADS, 
displayed when it is not available, is beneficial for the user. In fact, information on how long an automated driving function will be available increases acceptance and usability and positively influences the activation behavior because it helps in building the correct mental model in respect to the ADS for the specific context the driver finds themselves in [8]. A mental model is the representation a person has regarding how a certain device or system works $[9,10]$ and also contains assumptions about interdependencies of functions and reasons for the presence and absence of certain actions [11]. Therefore, assumptions on periods of availability of the automated vehicle and possible reasons for non-availability are also part of drivers' mental models. For example, if a driver takes the same route to work every day, they might be used to the ADS always being available in a specific highway segment. While the driver is in this segment, they might usually activate the automated driving function so that they can carry out some work. The segment where the ADS is available is part of the driver's mental model and therefore the question arises as to what would happen if the ADS were not available one day without obvious reasons? What if the ADS is available in a different segment than the one the driver is used to? This would lead to a discrepancy between the reality and the driver's mental model, which could lead to an increased mental workload [12,13]. Since mental models change with new experience, it would be adapted to this situation $[13,14]$, but as reasons for non-availability might not be static, this could change again. Therefore, it is important to help the user understand the ADS because feedback is essential in building a mental model and consequently for a successful human-machine interaction [15].

\subsection{Motivation}

The absence of availability might lead to automation surprise, which occurs when the behavior of the system is not congruent with the driver's expectations [16]. Unfulfilled expectations can also lead to emotions such as frustration [17], which should be avoided, since negative emotions can lead to a decrease in the driving performance [18]. It is hypothesized that drivers, when expecting availability based on experience, but faced by non-availability, search the aHMI [19] for an availability indicator. Furthermore, when drivers expect non-availability based on experience but instead encounter availability, the indication of availability might be overlooked if it is not designed to be sufficiently salient.

Hence, the aim of this study was to investigate how an HMI concept increasing transparency in a state of potential surprise due to a changed availability of the ADS influences the gaze behavior and the subjective rating of the automated driving function.

\subsection{Background: Context-Adaptive HMIs}

As described above, this paper presents a study investigating the effects an HMI concept has when a driver is potentially surprised by availability or non-availability of an automated driving function. In order for it to be possible for the driver to be surprised, they have to already have experience regarding the availability of the ADS on the road segment they are driving along and the current situation has to differ from those experiences. Since the context a person finds himself in is constituted by all the information available to describe the situation, the current road segment contributes to this context [20]. Therefore, the information displayed in the HMI dependent of the road segment and the availability of the ADS are context-adaptive, which is defined as explicit adaption of the behavior of a system to its context [21]. In more detail, a system that evaluates its behavior dependent on the historical inputs of the user, and then changes its behavior automatically if the evaluation results in the assumption that this change in behavior might increase the performance, is referred to as context-adaptive [22]. According to [23], there are different ways of adapting to a context: The context adaption of the presentation, which means the way a certain content is presented, differs depending on the context. One example is the automatic adaption of the display orientation [24]. Another possibility is adaption of the interaction based on past interaction patterns, which could cause a reduction of 
the interaction to a minimum [25]. Furthermore, it is possible to adapt the content to the context by hiding information that is irrelevant for a specific context [23].

With particular reference to driving, context can be defined as all the parameters describing a current situation with regard to the driver, the environment, and the vehicle [26]. Here, context-adaptive systems can be divided into two categories: Systems that conduct interactions for the user-for example, the system can automatically switch on the lights when it gets dark - and systems that support the user by providing information [27]. A typical example for context-adaptive systems is a recommendation to drive to the next gas station when the fuel level is low [28]. Context adaption is also used to increase safety while driving by explicitly not displaying unnecessary information in states of high workload or complex driving situations [29,30]. Ref. [27] implemented and evaluated an adaptive system that makes recommendations to the driver dependent on past behavior in specific driving contexts and found an increase in usability, also postulating improved acceptance.

Where the adaptivity of systems is concerned, it is possible to distinguish between four different types of behavior [31]. Firstly, there is non-context-adaptive behavior, which means that the system only exhibits behavior dependent on the user's input without any influence of the environment or context. The second type is non-context-transparent adaptive behavior. This behavior is dependent on the user's input and the environment but without exhibiting any interaction between the driver and the environment. The third behavior type, transparent context-adaptive behavior, is exhibited when the behavior depends on the user and the environment and the user is able to perceive the influence of the environment. The last behavior type, controlled context-adaptive behavior means that the system is dependent on the user and the environment and, furthermore, the user is able to influence the environment [31]. The case of adaptive availability notifications can be categorized as the third behavior type, transparent context-adaptive behavior, because the driver is able to perceive the environment; for example, a specific road segment.

As mentioned before, context-adaptive functions can have a positive influence on usability and acceptance [27]. Usability is the degree to which a user can perform a certain task with effectiveness, efficiency and satisfaction [32], while acceptance is defined as the willingness of a user to use a system when it is available [33]. According to the Technology Acceptance Model [34], acceptance consists of two dimensions, perceived ease of use and perceived usefulness, which are predictors for the intentions to use and therefore the actual usage behavior. This model was extended adding even more factors [35] and investigated in respect to conceptual relations to the construct of usability, showing unclear correlations [36]. Both constructs, usability and acceptance, are seen to be important evaluation criteria for HMIs [37].

\section{Research Objectives and Hypotheses}

The aim of this research paper was to transfer the method of the context-adaptive HMI to the case of availability notifications and to investigate it by means of a driving simulator study. If the user of an SAE Level 3 automation was used to a route and knows when it is possible to enable the automated driving function, they might be surprised if the availability changes and they cannot use the ADS the way they are used to. In this situation, the driver might expect availability, based on experience with this route, but no availability indicator appears on the HMI. Non-availability without any explanatory note, especially when availability is expected, can lead to the belief that there is a malfunction within the automated driving system [4]. While there should always be a reason why an automated driving function might not be available, considering these reasons currently always involves a partial assumption about what actual system limitations will look like when SAE Level 3 automations are launched [38]. Nevertheless, there are reasons considered realistic from the current point of view that are not necessarily static or immutable over time, such as lane markings, sensor errors, weather conditions, or moving roadworks.

It was hypothesized that the driver, when entering the road segment where availability is expected, might simply wait for the availability indicator to appear and consequently 
search the HMI for it, resulting in gaze behavior that is more focused on the instrument cluster (IC) where the availability indicator is expected to appear. Moreover, expectations not being met in this situation might lead to frustration [17], while a system not working as expected might be considered as less useful, with usefulness being a dimension of acceptance [34]. Additionally, a system not working when it is expected to work might be perceived as less effective, which influences usability [32].

For this study, an automation HMI [19] concept was designed with the aim of reducing negative effects due to automation surprise, by transparently informing the driver about non-availability in a situation where availability is expected, which is referred to as context adaptivity.

Another case investigated in this study was when a driver enters a road segment where they have experienced non-availability in the past, and then suddenly experience availability. In this situation, the context-adaptive HMI could indicate that activation of the ADS in this segment is possible for this journey. This additional display in the HMI might lead to earlier recognition of the availability and therefore earlier activation of the ADS, resulting in increased efficiency and usefulness.

Therefore, the hypotheses for this study were:

Hypothesis 1. The acceptance ratings for the ADS are higher when a context-adaptive HMI is used rather than the standard HMI.

Hypothesis 2. The usability ratings for the automation HMI are higher when a context-adaptive HMI is used rather than the standard HMI.

Hypothesis 3. The frustration ratings are lower when availability is expected but does not occur when a context-adaptive HMI is used rather than the standard HMI.

Hypothesis 4. With the context-adaptive HMI, the participants gaze at the IC less frequently when compared to the standard HMI upon entering a road segment where availability is expected but does not occur.

Hypothesis 5. With the context-adaptive HMI, the participants activate the ADS faster when compared to the standard HMI if non-availability is expected but availability occurs.

\section{Materials and Methods}

\subsection{Study Design}

The study presented in this paper was conducted in the static driving simulator of the Chair of Ergonomics at the Technical University Munich. A within-factor design was used, which means that every participant experienced each HMI-concept. As described above, two concepts were compared, a standard HMI vs. a context-adaptive HMI. Thus, the HMI concepts served as the independent variable. The difference between these concepts was the context-adaptive availability notification, which occurred in the context-adaptive HMI but not in the standard HMI concept.

The participants were informed that they would drive the same route in the driving simulator five times. Every journey represented the route to work for one weekday. Hence, the first journey represented the journey to work on Monday, while journey five represented the journey to work on Friday. The route was the same for all journeys. Every journey consisted of a small segment leading to the highway, three visually distinguishable highway segments and a short rural road leading to a building declared as the workplace. The ADS was always available on one highway segment. Participants were instructed to activate the ADS when available and to conduct NDRAs of their own choice. Smartphone usage was allowed and additionally a tablet computer was installed in the driving simulator with videos on it participants could watch. On Monday, Tuesday, and Thursday, the ADS was available on the first highway segment, while on Wednesday and Friday the ADS was available on the third highway segment. The highway segments were designed to 
be distinguishable in order for the participants to get used to the availability patterns dependent of the segment. Consequently, in the Wednesday journey, the participants should be surprised experiencing non-availability on the first segment and availability on the third segment. One-half of the participants experienced the context-adaptive HMI on Wednesday and the standard HMI on Friday, and the other half experienced the standard HMI on Wednesday and the context-adaptive HMI on Friday, in order to avoid carry-over effects. The availability patterns are summarized in Table 1.

Table 1. Availability Patterns and Sequence of the Concepts.

\begin{tabular}{|c|c|c|c|c|c|}
\hline \multirow[b]{2}{*}{ Day } & \multicolumn{3}{|c|}{ Availability of ADS } & \multicolumn{2}{|c|}{ Concept } \\
\hline & $\begin{array}{c}\text { Highway } \\
\text { Segment } 1\end{array}$ & $\begin{array}{c}\text { Highway } \\
\text { Segment } 2\end{array}$ & $\begin{array}{c}\text { Highway } \\
\text { Segment } 3\end{array}$ & Group 1 & Group 2 \\
\hline Monday & Available & Not available & Not available & & \\
\hline Tuesday & Available & Not available & Not available & & \\
\hline Wednesday & Not available & Not available & Available & CA & Not CA \\
\hline Thursday & Available & Not available & Not available & & \\
\hline Friday & Not available & Not available & Available & Not CA & CA \\
\hline
\end{tabular}

The Monday journey served as an acclimatization drive to allow the participants to get used to the driving simulator. The participants filled out the questionnaires after the Wednesday and Friday journey, which were the experimental drives in this study. Furthermore, the gaze behavior was only investigated for Wednesday and Friday, since on these days the difference in the availability was assumed to come into effect.

\subsection{Procedure}

The participants were welcomed to the driving simulator and informed about the study and its procedure. Written consent was obtained, after risks that can emerge from driving in a simulator, such as nausea, and the possibility of withdrawing from the study without consequences had been outlined. The participants filled in the demographic questionnaire, asking for age, gender, experience with driving simulator studies, driving behavior, and attitudes towards automated driving as well as to adaptive systems. They were instructed about how the driving simulator and the automation work and were familiarized with the system during a journey of about eight minutes, which counted as the Monday journey. The ADS was available on the first highway segment, in order for the test persons to learn how to activate and deactivate the automated driving function as well as to get to know how requests to intervene (RtIs) work. The participants were instructed that each journey represented a drive to work, thus making it apparent that the route was the same for each round. As described in Section 3.1, participants drove a total of five times and filled in the questionnaires investigating the subjective ratings towards the system after the third and the fifth journey, rating the last experienced concept. This was followed by a short structured guideline interview that asked whether the participants were surprised by the availability pattern at some point during the study. Furthermore, pictures of the concepts were shown, followed by questions regarding the participants' preferences and suggestions for improvement. This completed the experiment and the test persons left the laboratory. The whole procedure took about 90 min per participant and was completed in one session. Due to the Covid-19 pandemic, all items touched by the participants were sterilized and the laboratory was ventilated for at least half an hour before the next participant arrived.

\subsection{Driving Simulator and Test Track}

The study was conducted in the fixed-base driving simulator at the Chair of Ergonomics at the Technical University Munich (see Figure 1). SILAB 6.0 from the Würzburg Institute for Traffic Sciences $\mathrm{GmbH}$ running at $240 \mathrm{~Hz}$ was used to program the routes and 
implement the HMI-concepts. The driving simulation took place at $60 \mathrm{~Hz}$. The ADS could be activated by pressing a certain button on the steering wheel while control could be taken back by either braking or pressing the same button again.

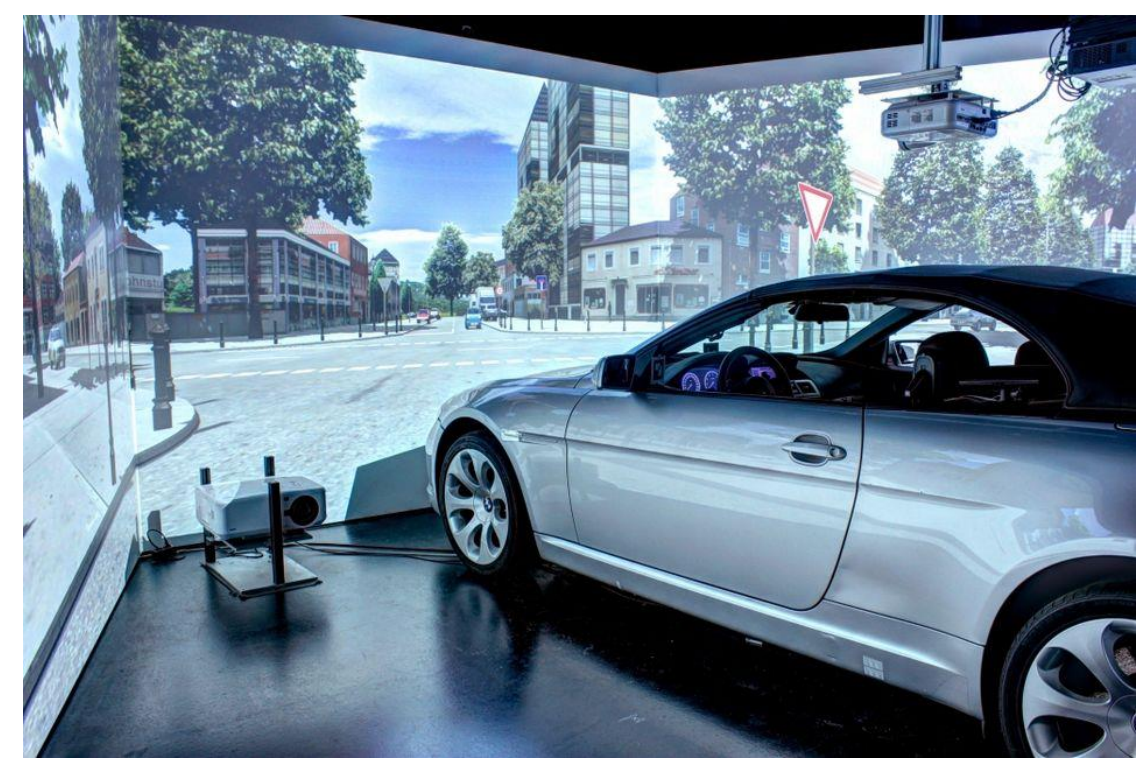

Figure 1. Driving simulator used for the study.

The three different highway segments were distinguishable for the participants due to the road design and the environment. Figure 2 shows the segments.

The remote eye-tracking system Smart Eye, based on three infrared cameras with a data collection rate of $60 \mathrm{~Hz}$, was used to investigate gaze behavior.
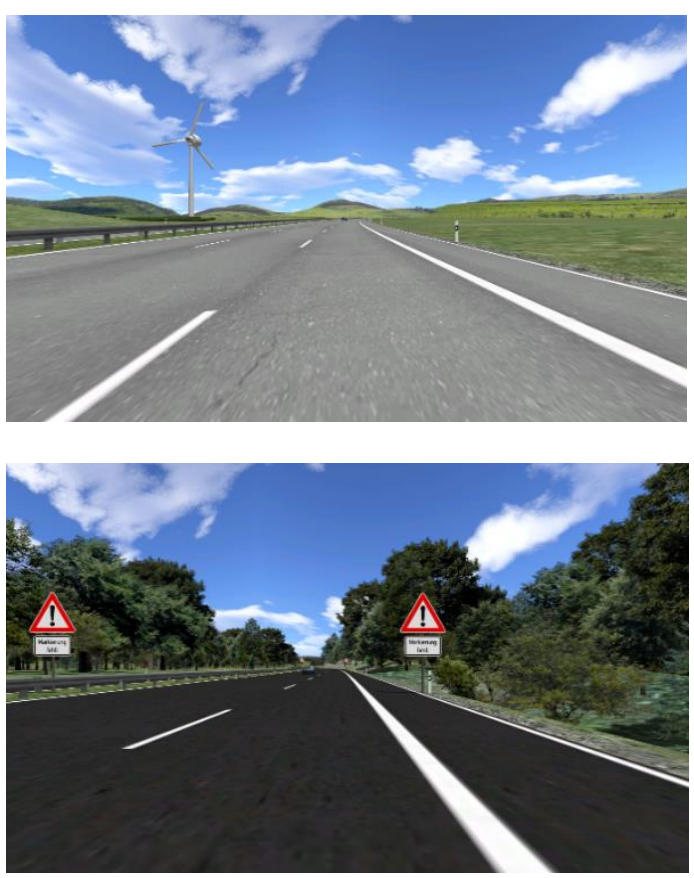

Figure 2. Cont. 


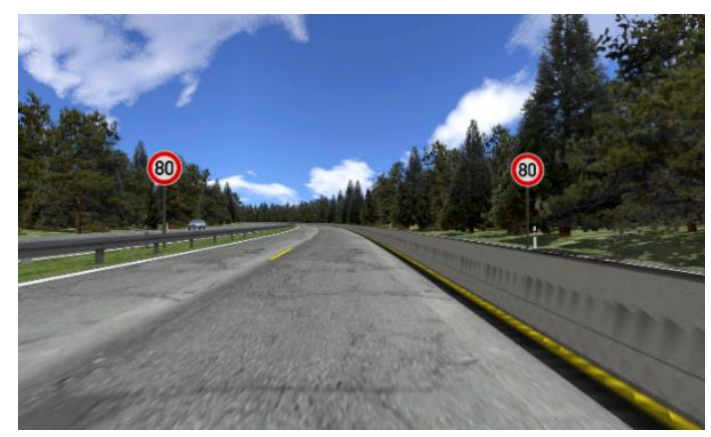

Figure 2. Distinguishable highway segments-here without traffic. The first picture shows the first highway segment, the picture in the middle the second, and the picture below shows the third highway segment.

\subsection{Human Machine Interface}

The two different HMI-concepts compared in this study differed only in respect to the context-adaptive availability notifications. Otherwise, both concepts included displays of the velocity, maximum speed, navigation, $\mathrm{rpm}$, and icons indicating the current system state and whether ADS was available as well as the availability duration when ADS was enabled $[15,39]$ (see Figure 2). In the event of non-availability, the ADS icon was dark gray, while it was white when ADS was available [40]. When ADS was active the display color was cyan (turquoise) [41]. The HMI design was based on [42] and was further developed for the purpose of this study.

The context-adaptive availability notifications consisted of a text displayed for $10 \mathrm{~s}$, stating "automation in this segment currently not available" in the event of unexpected nonavailability or "automation in this segment currently available" in the event of unexpected availability. After the notification text disappeared, an information icon appeared next to the automation icon. If participants pressed the information button on the steering wheel, a pop-up was displayed containing the same notification text. This was implemented in case participants missed the notification. The different displays are shown in Figure 3.

The HMI did not contain any other information or modalities than here described. An acoustical signal, for example, was not used for indicating availability since this would have changed the salience of the notification, which could have drawn more attention to the IC distorting the eye-tracking data.

\subsection{Dependent Variables and Measurement Methods}

The subjective measures used in this study were acceptance, usability, and frustration. Acceptance was measured using the van der Laan acceptance scale [43], consisting of nine items using semantic differentials to assess the attitude towards the system. Moreover, usability was measured using the system usability scale (SUS), consisting of 10 Likert-scale items [44] and frustration, as one dimension of the workload measure investigated with the NASA Raw Task Load Index (NASA-rTLX) [45].

To investigate whether participants looked at the IC more frequently when expecting availability but faced by an unexpected non-availability period, the attention ratio, gaze frequency, and gaze duration were analyzed for the first highway segment on Wednesdays and Fridays.

Another objective measure was the time until the participants activated the ADS in the event of unexpected availability, as it was hypothesized that participants might not recognize the availability when it was not expected. The time was measured from the appearance of the availability icon until activation of automation. 

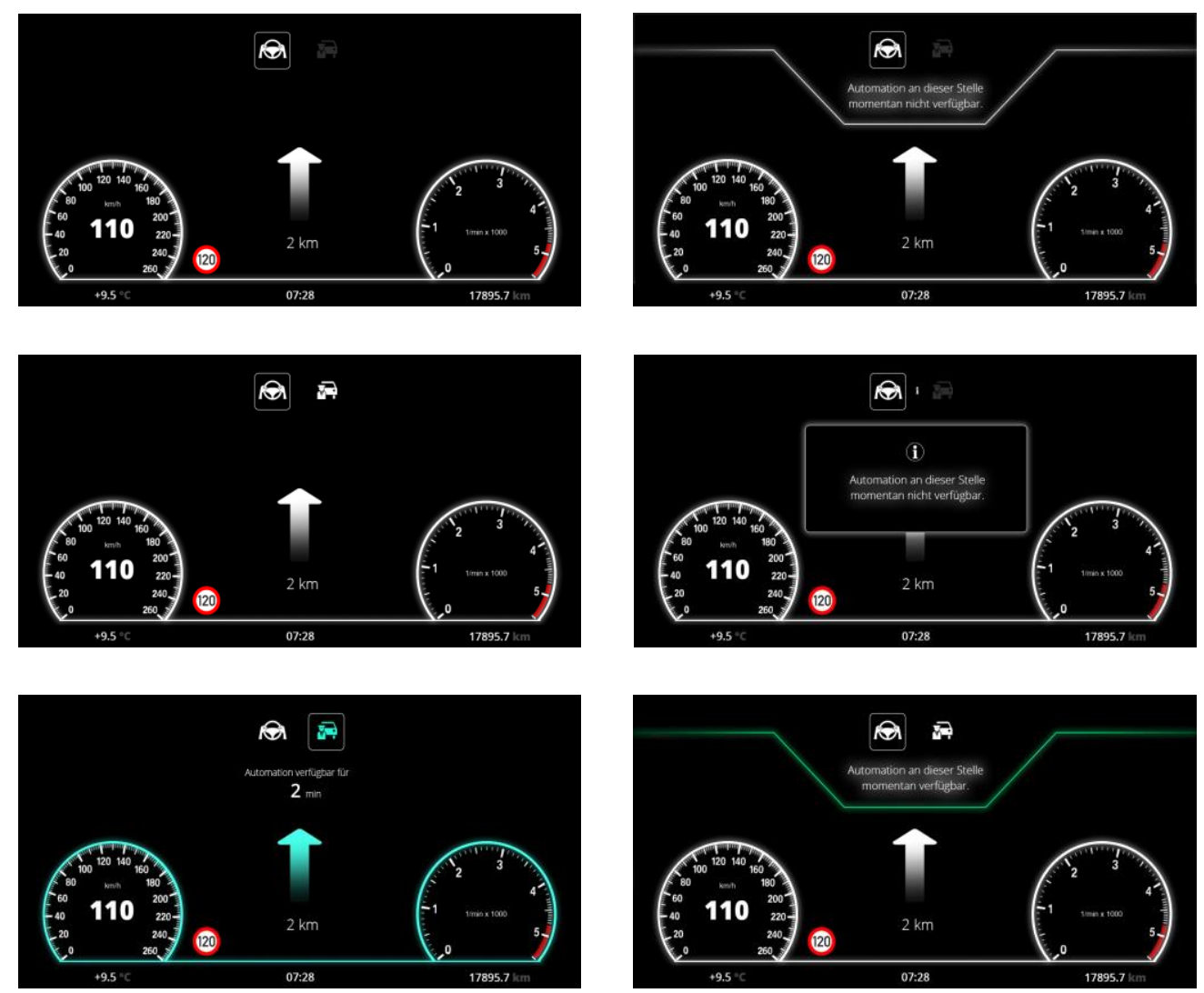

Figure 3. Human-machine interface (HMI) concepts: (Top left): Manual driving; (middle left): Automation available; (bottom left): Automation activated; (top right): Context-adaptive non-availability notification; (middle right): Pop-up with non-availability notification; (bottom right): Context-adaptive availability notification.

\subsection{Study Sample}

Thirty-five participants were recruited for this study. Five of the participants left the study due to simulation sickness. Therefore, 30 (including 10 female) participants finished the study and were included in the statistical analysis. The mean age was $M=28.5$ $(S D=3.9)$. Twenty-two participants stated that they study or work in a technical-related field. Eight participants had experience with driving simulator studies with a median of $M d n=7.5$ participations.

The Ethics Board of the Technical University Munich approved this study.

\subsection{Statistical Analysis}

The statistical analysis was conducted using RStudio. $t$-tests were conducted for the comparisons between the standard and context-adaptive HMI concept. If the presumptions were not fulfilled, the non-parametric alternative was chosen. Since the hypotheses were directional, one-tailed significance tests were used.

\section{Results}

\subsection{Subjective Data}

To test whether the acceptance rating of the participants differed dependent on the experienced HMI concept, a $t$-test for paired samples was conducted, after the ShapiroWilk test found no violation of the normal distribution assumption $(p>0.05)$. No effect was found $(t=0.26, p=0.41)$ for the comparison of the acceptance mean in the standard HMI condition $(M=0.86, S D=0.61)$ and the context-adaptive HMI condition $(M=0.90$; $S D=0.75)$.

Since the Shapiro-Wilk test revealed a violation of the normal distribution assumption for the mean differences of the SUS scores $(p<0.01)$, the non-parametric alternative to the 
$t$-test, the Wilcoxon test for paired samples was conducted. No significant effects were found $(z=-0.90, p=0.19)$ for the comparison of the SUS median for the standard HMI condition $(M d n=90)$ and the context-adaptive HMI condition $(M d n=88.75)$.

The Shapiro-Wilk test for the mean differences of the Frustration dimension of the NASA-rTLX indicated normal distribution $(p>0.05)$ and therefore a $t$-test for paired samples was conducted, finding no significant effects $(t=1.04, p=0.16)$. The means were $M=4.07(S D=4.14)$ for the standard HMI condition and $M=4.70(S D=5.17)$ for the context-adaptive condition.

To summarize these results, no effects regarding the subjective measures were found and therefore Hypotheses 1-3 had to be rejected. Means and standard deviations of the subjective data are summarized in Table 2.

Table 2. Means and Standard Deviations of the Subjective Ratings of Both HMI Concepts.

\begin{tabular}{ccc}
\hline Measure & Standard HMI & Context-Adaptive HMI \\
& $\boldsymbol{M}(\boldsymbol{S D})$ & $\boldsymbol{M}(\boldsymbol{S D})$ \\
\hline Acceptance (van der Laan) & $0.86(0.61)$ & $0.90(0.75)$ \\
Usability (SUS) & $87.33(13.37)$ & $84.42(15.78)$ \\
Frustration (NASA-rTLX) & $4.07(4.14)$ & $4.70(5.17)$ \\
\hline
\end{tabular}

\subsection{Objective Data}

Eye tracking data: For analyzing the eye tracking data, 3 participants had to be excluded due to data availability under 70\% [46].

No violations of the normal distribution assumption were found within the mean differences for the attention ratio $(p>0.05)$. The attention ratio, which means the ratio of the number of gazes at the IC, was higher for the context-adaptive HMI concept $(M=16.86 \%$, $S D=6.60 \%)$ than for the standard HMI concept $(M=14.11 \%, S D=6.54 \%)$. The $t$-test for paired groups showed a significant effect with a small effect size $(t=2.39, p=0.01, d=0.46)$.

For the gaze frequency, the Shapiro-Wilk test did not reveal a violation of the normal distribution assumption $(p>0.05)$. The $t$-test showed no significant effect $(t=1.02, p=0.16)$ for the comparison of the context-adaptive HMI concept $(M=0.30, S D=0.10)$ and the standard HMI concept $(M=0.28, S D=0.10)$.

The Shapiro-Wilk test for the mean differences of gaze durations directed at the IC also showed no violation to the normal distribution assumption $(p>0.05)$. It was higher for the context-adaptive HMI $(M=0.58, S D=0.17)$ than for the standard HMI concept $(M=0.52, S D=0.19)$ with a significant medium effect $(t=2.74, p<0.01, d=0.53)$.

Duration until activation: For analyzing the duration until activation, five participants had to be excluded, since they did not activate the automated driving function while driving on highway segment three. A Wilcoxon test was conducted, since the Shapiro-Wilk test revealed a violation of the normal distribution assumption $(p<0.01)$, to test the hypothesis that participants experiencing the standard HMI concept upon entering a highway segment where they expect non-availability take longer to recognize the unexpected availability leading to a longer period of non-activation. The time until activation after entering the third highway segment, on which the ADS was unexpectedly available, was compared for the context-adaptive HMI concept $(M d n=4.33, M=7.44, S D=7.58)$ versus the standard HMI concept $(M d n=5.10, M=14.78, S D=19.94)$. Since the normal assumption was violated according to the Shapiro-Wilk test $(p>0.05)$, a one-sided Wilcoxon test was conducted, showing no significant effect $(z=-0.90, p=0.18)$. The means and standard deviations of the objective measures are summarized in Table 3. 
Table 3. Means and Standard Deviation of the Objective Measures.

\begin{tabular}{ccc}
\hline Measure & $\begin{array}{c}\text { Standard HMI } \\
\boldsymbol{M}(\mathrm{SD})\end{array}$ & $\begin{array}{c}\text { Context-Adaptive HMI } \\
\boldsymbol{M}(\boldsymbol{S D})\end{array}$ \\
\hline Attention Ratio & $14.11 \%(6.54 \%)$ & $16.86 \%(6.60 \%)$ \\
Gaze Frequency & $0.28(0.10)$ & $0.30(0.10)$ \\
Gaze Duration & $0.52(0.19)$ & $0.58(0.17)$ \\
Duration until Activation & $14.78(19.94)$ & $7.44(7.58)$ \\
\hline
\end{tabular}

\subsection{Qualitative Data}

After they filled in the last questionnaires, the participants were interviewed using a semi-structured guideline interview. A total of $97 \%$ of the participants stated that they expected availability of the ADS on a segment where it was not available, while $86 \%$ stated that they expected non-availability on a segment where the ADS was available.

Regarding the standard HMI concept, $53 \%$ of the participants found the information displayed sufficient and $43 \%$ wished for notifications when availability changed unexpectedly. Thirty-seven percent of the participants wished for more salient icons and $10 \%$ for reasons explaining non-availability.

Regarding the context-adaptive HMI concept, $47 \%$ of the participants wondered about the notifications but $70 \%$ stated that they did not want them explained in the HMI. In addition, $70 \%$ stated that they found the information given in the context-adaptive HMI to be sufficient vs. $23 \%$ who did not find that enough information was displayed in the context-adaptive HMI concept. Thirteen percent of the participants stated that they found the notifications unnecessary and $47 \%$ wished for the reasons of non-availability to be additionally displayed. Fifty percent rated the notifications helpful in general.

Finally, the participants were asked to rate the two concepts on a 5-point Likert scale. The ratings of the context-adaptive HMI concept $(M d n=4.00, M=3.73, \mathrm{SD}=1.17)$ were generally higher than the rating of the standard HMI $(M d n=3.50, M=3.37, S D=0.89)$. The last question was which concept the participants preferred. Eighteen participants $(60 \%)$ stated that they preferred the context-adaptive HMI concept, 8 participants $(27 \%)$ stated that they preferred the standard concept, and 13\% could not decide which they preferred. The most prominent reason for preferring the context-adaptive HMI concept was the greater information content $(37 \%)$.

\section{Discussion and Limitations}

This study tested the hypotheses of whether context-adaptive availability notifications, which are displayed when the participants face unexpected (non-)availability of an automated driving function, improve the rating of the automated driving system and lead to less gazes at the IC in cases of unexpected non-availability and to faster activation in cases of unexpected availability.

As shown in Section 4, none of the hypotheses could be statistically confirmed. The results of the questionnaires showed similar ratings for both concepts for acceptance, usability, and frustration. No tendencies were revealed comparing the means, leading to the conclusion that the context-adaptive availability notification had no effect on the tested measures, even though the surprise in case of unexpected (non-)availability could be evoked, as shown in Section 4.3.

Where the eye tracking data were concerned, the effect found was contrary to the postulated one. The hypothesis was that participants, when expecting availability but facing non-availability, would search for an availability notification in the IC, where the availability was displayed in this study. This searching for the availability icon was postulated to lead to increased gazes at the IC in the standard HMI condition compared to the context-adaptive HMI condition, but the data showed that the participants actually took more gazes at the IC in the context-adaptive HMI condition. This leads to the conclusion that the reading of the context-adaptive notification text actually leads to more distraction than the postulated searching for an availability icon. Considering the increased distraction, 
the findings in this study did not indicate any safety benefits evoked by the context-adaptive HMI, which is not in accordance with the findings in the literature $[29,30]$. This can be explained by the different nature of the displays. In this study, the context-adaptivity led to additional notifications, while in the literature context-adaptivity was used to suppress information in order to decrease distraction in safety related situations [47].

The activation time in cases of unexpected availability of the ADS tended to agree with the hypothesis. Thus, participants activated the ADS faster when they did not expect availability when driving with the context-adaptive HMI concept. Since the information was the same in both concepts, this tendency can be explained by the higher salience of the availability in the context-adaptive HMI concept. Based on this explanation, the same effect could be achieved by giving an auditory signal when availability occurs. Moreover, it is assumed that this tendency would be diminished if the availability icon had been accompanied by an audible signal in both concepts in this study. Additionally, the standard deviation of the time until activation was substantially higher in the standard condition in comparison to the context-adaptive condition. This led to the assumption that less outliers occur with the more salient context-adaptive availability notifications, which means that less participants took longer than expected to recognize the availability.

The qualitative interview showed no clear preferences of participants, even though the majority stated that they preferred the context-adaptive HMI concept. Moreover, participants wished for reasons explaining non-availability which is in accordance with [4] and, furthermore, the participants stated that they desired more salient availability notifications. As explained above, this would probably lead to a decreased difference between the two concepts regarding the activation time.

Another limitation of this study was the rather homogenous sample. Participants mostly were students or employees of the Technical University Munich. A more heterogeneous sample with older participants and those without technical background could have led to different results or to a broader range of qualitative statements.

In summary, it can be concluded that the context-adaptive availability notifications can be beneficial for some users, especially when availability notifications are not salient, and should contain more information, for instance, reasons for non-availability or when the ADS will be available again [8]. Since some participants stated that they preferred the standard HMI, it should be possible for future users to disable the context-adaptive availability notifications. Furthermore, consideration should be given to designing them in a less distracting way. For instance, it could be annoying for the user if each availability were accompanied by an audible signal; however, instead the audible signal could replace the visual context-adaptive notification and therefore only occur when the (non-)availability is unexpected. When the ADS is unexpectedly not available, the context-adaptive notification could be complemented with the reason for non-availability, in order to increase the system transparency.

The similarity of the subjective ratings could be explained by the visual similarity of the two different HMI concepts. Their only difference was in respect to the context-adaptive notifications and, therefore, the questionnaires might not be sensitive enough to detect these differences, especially with regard to usability. Otherwise, it is also possible that the notifications simply do not affect the constructs acceptance and frustration.

The study design, which was simulating weekdays, might not have had the desired outcome-leading to a habituation effect regarding the availability pattern, which would explain the absent effects. Nonetheless, this is not likely because the participants stated in the qualitative interview that they had been surprised by the changed (non-)availability. Therefore, it can be concluded that the number of rides in this study was enough to allow the participants to build a mental model regarding the availability patterns. It could be shown that mental models were sufficiently built after five rides in a longitudinal study [48]. Since in this study participants drove five times in a row on one day, the mental model might have been formed faster, which would explain why the habituation to the availability patterns was successful. 
The most prominent reason for preferring the context-adaptive HMI concept was the greater information content. This reason is based on an objectively wrong assumption, since the information content was the same in both concepts. The context-adaptive notifications only said in words what the icons in the standard HMI concept conveyed. This could lead to the conclusion that the participants might not have understood the principle of adaptivity in this study. Using a study design in which the context adaptivity occurs more often, while habituation is established as in this study, could help the participants to understand this principle. Therefore, future studies should investigate whether a difference in the information content-existent or imagined-influences the ratings of context-adaptive HMIs. Furthermore, research should analyze if availability notifications should be designed more salient in general or if context-adaptivity can help in situations in which users might oversee specific information because they do not expect them. Moreover, it should be investigated if more realistic travel durations in combination with a longitudinal study design could have an impact on the results of this kind of research. Another important aspect with respect to context-adaptivity are potential consequences of false assumptions of the system. Future studies should investigate how users react if the system adapts wrongly or fails to adapt when this is expected.

Author Contributions: Conceptualization, S.D., A.F. and C.M.; methodology, S.D. and A.F.; software, C.M. and A.F.; formal analysis, S.D.; investigation, C.M.; writing-original draft preparation, S.D.; writing-review and editing, A.F., K.B. and S.D.; visualization, S.D.; supervision, K.B. All authors have read and agreed to the published version of the manuscript.

Funding: This research was funded by AUDI AG.

Institutional Review Board Statement: The study was conducted in accordance with the guidelines of the Declaration of Helsinki, and approved by the Ethics Committee of Technical University Munich (approval code: $486 / 20$ S).

Informed Consent Statement: Informed consent was obtained from all subjects involved in the study.

Data Availability Statement: The data presented in this study are available on request from the corresponding author. The data are not publicly available due to privacy restrictions.

Conflicts of Interest: The authors declare no conflict of interest.

\section{References}

1. Society of Automotive Engineers. Taxonomy and Definitions for Terms Related to Driving Automation Systems for On-Road Motor Vehicles (J3016_201806); SAE: Warrendale, PA, USA, 2018.

2. Howard, D.; Dai, D. Public Perceptions of Self-driving Cars: The Case of Berkeley, California. In Proceedings of the 93rd Annual Meeting TRB, Washington, DC, USA, 19 December 2013.

3. Schoettle, B.; Sivak, M. Public Opinion about Self-Driving Vehicles in China, India, Japan, the US, the UK, and Australia; University of Michigan Transportation Research Institute: Ann Arbor, MI, USA, 2014.

4. Danner, S.; Pfromm, M.; Limbacher, R.; Bengler, K. Information needs regarding the purposeful activation of automated driving functions-An exploratory study. In Proceedings of the Human Factors and Ergonomics Society Europe Chapter 2019 Annual Conference, Nantes, France, 2-4 October 2019.

5. Pfleging, B.; Rang, M.; Broy, N. Investigating User Needs for Non-Driving-Related Activities During Automated Driving. In Proceedings of the 15th International Conference on Mobile and Ubiquitous Multimedia-MUM '16, Rovaniemi, Finland, 12-15 December 2016; Häkkila, J., Ojala, T., Eds.; ACM Press: New York, NY, USA, 2016; pp. 91-99, ISBN 9781450348607.

6. Schoettle, B.; Sivak, M. A Survey of Public Opinion about Autonomous and Self-Driving Vehicles in the USA, the UK, and Australia; Transportation Research Institute (UMTRI): Ann Arbor, MI, USA, 2014.

7. Hecht, T.; Feldhütter, A.; Draeger, K.; Bengler, K. What do you do? An analysis of non-driving related activities during a 60 minutes conditionally automated highway drive. In Human Interaction and Emerging Technologies; Ahram, T., Taiar, R., Colson, S., Choplin, A., Eds.; Springer International Publishing: Cham, Switzerland, 2020; pp. 28-34, ISBN 978-3-030-25628-9.

8. Danner, S.; Pfromm, M.; Bengler, K. Does Information on Automated Driving Functions and the Way of Presenting It before Activation Influence Users' Behavior and Perception of the System? Information 2020, 11, 54. [CrossRef]

9. Norman, D.A. Some observations on mental models. In Readings in Human-Computer Interaction: A Multidisciplinary Approach; Baecker, R.M., Buxton, W.A.S., Eds.; Kaufmann: Los Altos, CA, USA, 1987; pp. 241-244, ISBN 0934613249.

10. Kieras, D.E.; Bovair, S. The Role of a Mental Model in Learning to Operate a Device. Cogn. Sci. 1984, 8, 255-273. [CrossRef] 
11. Kazi, T.A.; Stanton, N.A.; Walker, G.H.; Young, M.S. Designer driving: Drivers' conceptual models and level of trust in adaptive cruise control. Int. J. Veh. Des. 2007, 45, 339. [CrossRef]

12. Zwaan, R.A.; Radvansky, G.A.; Hilliard, A.E.; Curiel, J.M. Constructing Multidimensional Situation Models during Reading. Sci. Stud. Read. 1998, 2, 199-220. [CrossRef]

13. Beggiato, M.; Krems, J.F. The evolution of mental model, trust and acceptance of adaptive cruise control in relation to initial information. Transp. Res. Part F Traffic Psychol. Behav. 2013, 18, 47-57. [CrossRef]

14. Stanton, N.A.; Young, M.S. A proposed psychological model of driving automation. Theor. Issues Ergon. Sci. 2000, 1, 315-331. [CrossRef]

15. Beggiato, M.; Hartwich, F.; Schleinitz, K.; Krems, J.; Othersen, I.; Petermann-Stock, I. What would drivers like to know during automated driving? Information needs at different levels of automation. In Proceedings of the 7th Conference on Driver Assistance, Lyon, France, 21 April 2015. [CrossRef]

16. Carsten, O.; Martens, M.H. How can humans understand their automated cars? HMI principles, problems and solutions. Cogn. Technol. Work. 2018, 21, 3-20. [CrossRef]

17. Ochs, M.; Pelachaud, C.; Sadek, D. An Empathic Virtual Dialog Agentto Improve Human-Machine Interaction. In Proceedings of the 7th International Joint Conference on Autonomous Agents and Multiagent Systems; Padgham, L., Ed.; International Foundation for Autonomous Agents and Multiagent Systems: Cambridge, MA, USA, 2008; Volume 1, pp. 89-96, ISBN 9780981738109.

18. Braun, M.; Schubert, J.; Pfleging, B.; Alt, F. Improving Driver Emotions with Affective Strategies. Multimodal Technol. Interact. 2019, 3, 21. [CrossRef]

19. Bengler, K.; Rettenmaier, M.; Fritz, N.; Feierle, A. From HMI to HMIs: Towards an HMI Framework for Automated Driving. Information 2020, 11, 61. [CrossRef]

20. Abowd, G.D.; Abowd, G.D.; Dey, A.K.; Dey, A.K.; Brown, P.J.; Brown, P.J.; Davies, N.; Davies, N.; Smith, M.E.; Smith, M.E.; et al. Towards a better Understanding of Context and Context-Awareness. In Proceedings of the International Symposium on Handheld and Ubiquitous Computing, Karlsruhe, Germany, 27-29 September 1999; Springer: Berlin/Heidelberg, Germany, 1999; pp. 304-307.

21. Fahrmair, M.R. Kalibrierbare Kontextadaption für Ubiquitous Computing. Master's Thesis, Technische Universität München, München, Germany, 2005.

22. Garzon, S.R. Kontextsensitive Personalisierung Automotiver Benutzerschnittstellen: Entwicklung und Anwendung eines Regelbasierten Verfahrens zur Erkennung Situationsabh ängiger Mensch-Maschine-Interaktionen. Master's Thesis, Technischen Universität Berlin, Berlin, Germany, 2013.

23. Jöst, M. Adapting to the user. In Ubiquitous and Pervasive Computing: Concepts, Methodologies, Tools, and Applications; Symonds, J., Ed.; Information Science Reference: Hershey, PA, USA, 2010; pp. 1006-1020, ISBN 9781605669601.

24. Schmidt, A.; Beigl, M.; Gellersen, H.-W. There is more to context than location. Comput. Graph. 1999, 23, 893-901. [CrossRef]

25. Cheverst, K.; Davies, N.; Mitchell, K.; Efstratiou, C. Using Context as a Crystal Ball: Rewards and Pitfalls. Pers. Ubiquitous Comput. 2001, 5, 8-11. [CrossRef]

26. Hoch, S. Kontextmanagement und Wissensanalyse imkognitiven Automobil der Zukunft. Ph.D. Thesis, Technische Universität München, München, Germany, 2008.

27. Walter, N. Personalization and Context-Sensitive User Interaction of In-Vehicle Infotainment Systems. Master's Thesis, Technische Universität München, München, Germany, 2018.

28. Bader, R.; Wörndl, W.; Prinz, V. Situation Awareness for Proactive In-Car Recommendations of Points-Of-Interest (POI). 2010. Available online: https: / www.semanticscholar.org/paper/Situation-Awareness-for-Proactive-In-Car-of-(-POI-)-Bader-W\% C3\%B6rndl/bebed06ad1d664f6c1fb424efb523c161b6787d3 (accessed on 26 March 2021).

29. Rosario, B.; Lyons, K.; Healey, J. A Dynamic Content Summarization System for Opportunistic Driver Infotainment. In Proceedings of the 3rd International Conference on Automotive User Interfaces and Interactive Vehicular Applications, Salzburg, Austria, 30 November-2 December 2011; Tscheligi, M., Ed.; ACM: New York, NY, USA, 2011; p. 95, ISBN 9781450312318.

30. Amditis, A.; Andreone, L.; Pagle, K.; Markkula, G.; Deregibus, E.; Rue, M.R.; Bellotti, F.; Engelsberg, A.; Brouwer, R.; Peters, B.; et al. Towards the Automotive HMI of the Future: Overview of the AIDE-Integrated Project Results. IEEE Trans. Intell. Transp. Syst. 2010, 11, 567-578. [CrossRef]

31. Broy, M.; Leuxner, C.; Sitou, W.; Spanfelner, B.; Winter, S. Formalizing the Notion of Adaptive System Behavior. In Proceedings of the 24th Annual ACM Symposium on Applied Computing 2009, Honolulu, HI, USA, 8-12 March 2009; Shin, S.Y., Ossowski, S., Eds.; ACM: New York, NY, USA, 2009; p. 1029, ISBN 9781605581668.

32. International Organization for Standardization. Ergonomics of Human-System Interaction-Part 11: Usability: Definitions and Concepts, 2018 (ISO 9241-11); ISO: Geneva, Switzerland, 2018.

33. Adell, E.; Várhelyi, A.; Nilsson, L. The Definition of Acceptance and Acceptability. In Driver Acceptance of New Technology; Apple Academic Press: Palm Bay, FL, USA, 2018; pp. 11-22, ISBN 9781315578132.

34. Davis, F.D. Perceived Usefulness, Perceived Ease of Use, and User Acceptance of Information Technology. MIS Q. 1989, 13, 319-340. [CrossRef]

35. Venkatesh, V.; Davis, F.D. A Theoretical Extension of the Technology Acceptance Model: Four Longitudinal Field Studies. Manag. Sci. 2000, 46, 186-204. [CrossRef] 
36. Lin, C.-C. Exploring the relationship between technology acceptance model and usability test. Inf. Technol. Manag. 2013, 14, 243-255. [CrossRef]

37. François, M.; Osiurak, F.; Fort, A.; Crave, P.; Navarro, J. Automotive HMI design and participatory user involvement: Review and perspectives. Ergonomics 2016, 60, 541-552. [CrossRef]

38. Schömig, N.; Wiedemann, K.; Naujoks, F.; Hergeth, S.; Keinath, A.; Neukum, A. How important is the plausibility of test scenarios within usability studies for AV HMI? In Advances in Human Aspects of Transportation; Stanton, N., Ed.; Springer International Publishing: Cham, Switzerland, 2020; pp. 77-84. ISBN 978-3-030-50942-2.

39. Feierle, A.; Danner, S.; Steininger, S.; Bengler, K. Information Needs and Visual Attention during Urban, Highly Automated Driving-An Investigation of Potential Influencing Factors. Information 2020, 11, 62. [CrossRef]

40. Feierle, A.; Holderied, M.; Bengler, K. Evaluation of Ambient Light Displays for Requests to Intervene and Minimal Risk Ma-neuvers in Highly Automated Urban Driving. In Proceedings of the 2020 IEEE 23rd International Conference on Intelligent Transportation Systems (ITSC), Rhodes, Greece, 20-23 September 2020; IEEE: New York, NY, USA, 2020; pp. 1-8, ISBN 978-17281-4149-7.

41. Muthumani, A.; Diederichs, F.; Galle, M.; Schmid-Lorch, S.; Forsberg, C.; Widlroither, H.; Feierle, A.; Bengler, K. How visual cues on steering wheel improve users' trust, experience, and acceptance in automated vehicles. In Advances in Human Aspects of Transportation; Stanton, N., Ed.; Springer International Publishing: Cham, Switzerland, 2020; pp. 186-192, ISBN 9783030509422.

42. Feierle, A.; Bücherl, F.; Hecht, T.; Bengler, K. Evaluation of display concepts for the instrument cluster in urban automated driving. In Human Systems Engineering and Design II; Ahram, T., Karwowski, W., Pickl, S., Taiar, R., Eds.; Springer International Publishing: Cham, Switzerland, 2020; pp. 209-215, ISBN 9783030279271.

43. Van Der Laan, J.D.; Heino, A.; De Waard, D. A simple procedure for the assessment of acceptance of advanced transport telematics. Transp. Res. Part C Emerg. Technol. 1997, 5, 1-10. [CrossRef]

44. Brooke, J. SUS: A “quick and dirty" usability scale. In Usability Evaluation in Industry; Jordan, P.W., Thomas, B., McClelland, I.L., Weerdmeester, B., Eds.; Chapman and Hall/CRC: Boca Raton, FL, USA, 1996; ISBN 9780748404605.

45. Hart, S.G. Nasa-task load index (NASA-TLX); 20 years later. Proc. Hum. Factors Ergon. Soc. Annu. Meet. 2006, 50, 904-908. [CrossRef]

46. International Organization for Standardization. Road Vehicles-Measurement of Driver Visual Behaviour with Respect to Transport Information and Control Systems_Part 2: Equipment and Procedures. ISO/TS 15007-2:2014; ISO: Geneva, Switzerland, 2014.

47. Köhler, L.; Bengler, K.; Mergl, C.; Maier, K.; Wimmer, M. Validation of a Telephone Manager for stressful driving situations. In Proceedings of the Human Factors and Ergonomics Society Europe Chapter 2014 Annual Conference: Human Factors in High Reliability Industries, Lisbon, Portugal, 8-10 October 2014; de Waard, D., Sauer, J., Röttger, S., Kluge, A., Manzey, D., Weikert, C., Toffetti, A., Wiczorek, R., Brookhuis, K., Hoonhout, J., Eds.; HFES: Santa Monica, CA, USA, 2015.

48. Beggiato, M.; Pereira, M.; Petzoldt, T.; Krems, J. Learning and development of trust, acceptance and the mental model of ACC. A longitudinal on-road study. Transp. Res. Part F Traffic Psychol. Behav. 2015, 35, 75-84. [CrossRef] 